\title{
An Architectural Model on Employment Opportunities as Aftercare Program
}

\author{
Neriza V. Bustillo, Thelma D. Palaoag, Joseph Kristian Reyes
}

\begin{abstract}
The aftercare program of a drug rehabilitation center is a continuing treatment receive by ex-drug abusers immediately after being discharged from a residential rehabilitation center. This program caters services that help in the development of skills of ex-drug abusers as they move out from the facility of the rehabilitation center for them earn for living. The rehabilitation centers offer opportunities for employment as they allow other agencies to offer job and training and seminar services for ex-drug abusers. In the implementation of the job opportunities as an aftercare program, the selection of ex-drug abuser who is ready to work, ready to be trained, and ready for a further referral is a crucial decision. To this end, an architectural model that can match ex-drug users profile and relevant jobs, training, and referrals were needed. The developed architecture will serve as their basis for a decision support system development.
\end{abstract}

Rundown phrases- architectural model; employment opportunities; ex-drug abusers.

\section{I.INTRODUCTION}

Drug addiction is rampant and accessible to all, leaving the young population easy victims. It is a worldwide phenomenon that generates problems not only within families but to society. This inability to control oneself to use the illicit drug can lead to harming anyone which usually cause problems in family structure, and to the society. Rehabilitation center services enable people to counteract addiction's powerful, disturbing effects on their brain and behavior and recover control of their lives. It is not easy to cure, but like other chronic diseases, addiction can be managed successfully [1]. It is in this premise why the government in all countries worldwide provides rehabilitation and treatment to this at-risk population.

In the Philippines, there is an increase in the number of drug dependents. As of March 2017, the Philippine Drug Enforcement Agency (PDEA) stated that there were around 4.7 million illicit drug users [2] and in the National Capital Region alone, $92 \%$ of barangays were affected [3]. In the implementation of the Operation Double Barrel' or Oplan Tokhang, there were 1.18 million illicit drug users who have surrendered [4] with an additional 44,070 arrested and 2,206 fatalities [5]. It is because of this increasing figure that the government, other organizations, religious, and individuals have come up with aftercare services meant for behavior modification and integration into society through livelihood programs.

Revised Manuscript Received on July 18, 2019.

Neriza V. Bustillo, College of IT Education, University of Pangasinan, Dagupan City, Philippines. (E-mail: nvbustillo@up.phinma.edu.ph)

Thelma D. Palaoag, University of the Cordilleras, Baguio City, Philippines (E-mail: tpalaoag@gmail.com)

Joseph Kristian Reyes, Department of Health Drug Treatment and Rehabilitation, Center - Region I, Dagupan City, Philippines (E-mail: jkbreyes@gmail.com)
According to [6], approximately 275 million people globally use drugs at least once a year. Roughly $5.6 \%$ of the global populations aged 15 to 64 years old were using illicit drugs. The associated harm is highest among young ones compared to older people. Most researches claimed that the critical risk period for the initiation of illicit drug use is adolescence with age 15 to 17 years old. Cannabis was the most widely consumed drug in 2016. The majority of drug users are men.

Last 2017, a report was released stating that $5 \%$ of the World's population that have pursued the help of rehabilitation centers for behavioral reforms were on the age bracket of 15 to 64 years old[7]. Over the years, these rehabilitation centers have admitted drug addicts to help them recover from drug misuse through rehabilitation programs. In the Philippines alone, among 53 licensed drug abuse treatment and rehabilitation centers, there were only 15 centers operated by the Department of Health. A total of 8,662 graduate rehabilitation residents have completed the rehabilitation program from 2016 to July 2018. Still, there are 8,826 remain as an inpatient while there were around 5,450 as aftercare patients [8]. The opportunity for behavior modification is the ultimate goal of drug rehabilcenters. Their program offers services for ex-drug abusers to overcome psychological, physical, and emotional complications [9].

\section{Aftercare Program}

In recent years, all government agencies have focused their attention on the recovery program. It was driven mainly by extensive research in the United States of America (USA), and reports by people who have overcome drug addiction who were promoting the idea that recovery is probable. An aftercare program is an essential aspect of rehabilitation treatment for ex-drug abusers for them to maintain abstinence after being discharged from the rehabilitation centers' inpatient program [10]. Aftercare Program (ACP) helps drug-dependent persons to adopt in the everyday community life and provide opportunities to look into critical issues that go along with abstinence and recovery. Aftercare provides a safe environment for continued support until it is no longer needed. It also aims to impart new skills for sustaining recovery such as handling everyday work and duties; managing family problems, peer issues, and workplace; getting more friends; reinstate to work referrals to agencies that facilitate employment 
opportunity and livelihood program, and developing newwisdom into the ex-drug abusers psychological and emotional well-being. [11].

Employment can help to reinforce and sustain the recovery process [12]. The inclusion, esteem, and opportunities for social contact, which are associated with employment, also help in preventing lapse and relapse [13]

[14]. Employment can help in the attainment of the objective of the aftercare program in providing services that will increase the self-reliance and skills of ex-drug abusers. It is possible through educational, vocational, and social programs offered by other agencies supporting the aftercare program of the rehabilitation center [11].

Drug abuse treatment and rehabilitation centers have recognized the necessity for recovery as a fundamental component of the services offered by the rehabilitation center. The government has also come up with strategies to address substance misuse. This two agencies shifted the treatment to a community-based treatment and training and employment opportunities that can help them in their new life.

\section{Decision Support Systems}

Decision Support Systems (DSS) are information systems used by business and organizational decision-makers as they effectively perform their decision-making activities. Specifically, it is intended to help decision-makers to gather the information that can be used to identify and solve problems for sound decision making [15]. Aside from decision support systems, it also includes data warehousing and analytical processing systems [16].

There are many classifications associated with the decision support system. First is the text-oriented DSS that deals with the process of document creation, revision, and reviews. Followed by the database-oriented DSS that deals with the organization of the database, query capabilities, and generation of reliable reports. Then the spreadsheet-oriented DSS that allows the development of models to execute DSS analysis. Also, solver-oriented DSS uses a solver to solve a particular problem. Furthermore, the rule-oriented DSS that uses the knowledge component such as procedural and inferential rules. Lastly, the compound DSS includes the combination of two or more classifications of DSS [17].

Furthermore, a DSS is successful if it is simple, fast, and easy to communicate with the other systems involved in the business process. Typically, it is consists of a language system which served as a mechanism in the communication with stakeholders and other components of the system; a knowledge system which served as the problem domain knowledge repository of existing DSS; and a problem processing system that performs the problem manipulation capabilities which are necessary for decision-making. Meanwhile, the decision-making process is consists of three phases, namely: intelligence, design, and choice. The intelligence phase is the process of tracing and detecting the scope and recognition of the problems. The design phase is the process of discovery, development, and analysis of alternative course actions. It can be done by understanding the problem, creating a solution, and testing the feasibility of the solution. Lastly, the choice phase is the process of selecting alternative actions that can be executed [17].

The primary purpose of this study is the formulation of the architectural model in the design and development of a DSS for the aftercare program of Drug Rehabilitation Center.

\section{METHODOLOGY}

A qualitative type of research, specifically descriptive research design, was utilized in the conduct of the study. The researcher used observation and interviews to determine the existing infrastructure in the rehabilitation center and to gather pertinent information in the conduct of the study. Document analysis of different research findings was conducted to develop the architectural model. The system architecture of [18] [19] served as the basis in the development of the architectural model of the Aftercare system.

\section{DISCUSSION \& RESULTS}

The study used the 3-tier architecture and Model-ViewController (MVC). These two architectures are known to be very useful to decouple and isolate various components of a more extensive system. In the 3-tier architecture, the presentation layer takes in-charge in coordinating the retrieval and modification of the model layer, the business layer represents the business rules translated in programming codes, and the data access layer retrieves and access pertinent data stored in the database. Also, the business layer accepts the request from the users through the presentation layer, then processes the request or queries to the database server. The filtered data is from the database server is presented to the end-users through the presentation layer. Data integrity, scalability, and security are key features of the 3-tier architecture that makes it advantageous with the other architectures [20].

In the MVC, the model layer represents the entirety of the system or application, the view layer represents the user interface, and the controller layer represents the programming code that responds to the user and the changes in the model layer. According to [21], MVC helps decouple data access and business logic from its presentation to the user. Figure 1 shows the 3-tier architecture is composed of the presentation layer, business layer, and data access layer, while Figure 2 shows the MVC is composed of the model, view, and controllers.

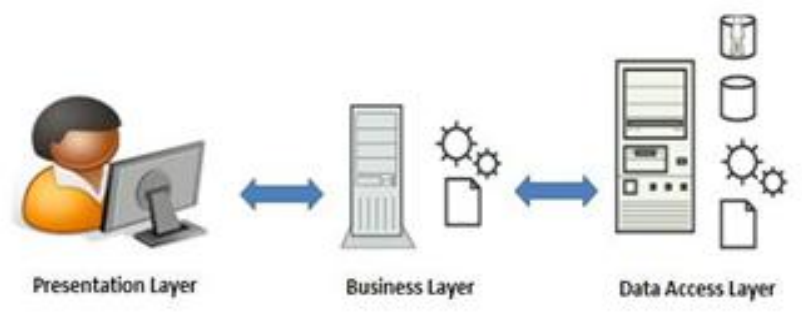

Figure 1. 3-tier Architectu 


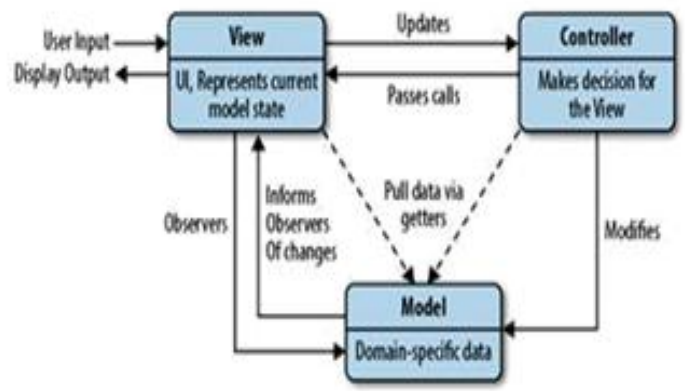

Figure 2. MVC

The 3-tier architecture and MVC were utilized to model the accessibility of system and data processing. The architecture serves as the foundation of the developed architecture model for the Aftercare program decision support system. The architecture model in Figure 3 was composed of the following main components: Ex-drug abusers (EDA) Search Processor, EDA Profile matcher, and Batch processor.

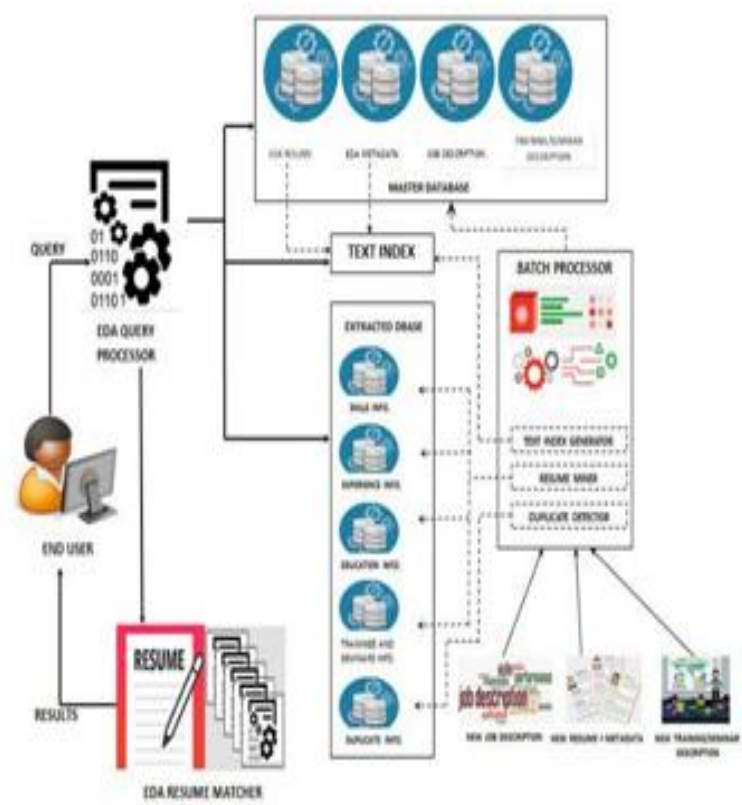
Figure 3. Aftercare Employment Opportunities
Decision Support System Architecture

\section{EDA Search Processor}

The EDA search processor interacts with various components of the architectures such as the text index, database, and EDA Profile Matcher. On the process, the end-user input the string chose the search criteria, and then returned a ranked list of EDA based on the search criteria. A

The EDA search processor interacts with various components of the architectures such as the text index, database, and EDA Profile Matcher. On the process, the end-user input the string chose the search criteria, and then returned a ranked list of EDA based on the search criteria. A

search parser is associated with it that parses the search strings and converts it to a format suitable for the need of the Aftercare department. The text search moved to the text index and database. It is on the database where the EDA

search processor extracts the data required. The obtained results were collected and submitted to EDA Profile Matcher.

\section{EDA Profile Matcher}

The EDA profile matcher is the main element of the Aftercare decision support system. The process begins when the EDA search processor searches the master database and extracted database using an innovative matching method to retrieve the most applicable employment opportunities and training/seminars opportunities suitable to the EDA profile. A sorted list of employment opportunities and training/seminars opportunities suited to the EDA profile is returned and displayed to the end-users through the EDA search interface. It is also the element that organizes the EDA profile list based on the extent of the profile match with the job opportunity description and training/seminars opportunities description. In the end, the application displayed the sorted list of EDA profiles, job opportunities, and training/seminars opportunities to the end-user.

\section{Batch Processor}

This component processes all EDA profile. It

is

composed of the following modules [18]:

1. The text index which is capable of performing a full-text search on EDA profiles. A text search engine library is used to perform the full-text search over EDA profiles.

2. The profile miner is the core element of Aftercare Decision Support System architecture. It performs data normalization and data consistency checks then fetched the data in the extracted database. The profile miner is the EDA profile extraction system, which is composed of an extractor, datanormalization, and data consistency checker.

3. The duplicate detector does the detection of multiple application of EDA to available job opportunities and training/seminar opportunities. It is also used toidentify duplicate job opportunities and training/seminar opportunities and to identify similar EDA profiles. The system provides reports on duplicate EDA profiles, job opportunities, and training/seminar opportunities

The architectural model is very much suitable for the existing business process and infrastructure of the rehabilitation center. In the full implementation of the architecture model, the aftercare head and staff and all other stakeholders involved should be properly oriented on how to use the decision support system. An interface where the exdrug abuser can input their information, job experience, and educational attainment are very much essential in the DSS. It serves as the raw data that will be processed using the data mining algorithm. The Resume miner normalized and checked for inconsistencies of the raw data and stores it in the database server. A database server is where these data are stored and process. It is also where the skill, resume, training, and seminar matching happens using matching algorithms. 


\section{CONCLUSIONS}

The architecture model appropriate to EDA information that needs security and confidentiality is the 3-tier architecture and the Model-View-Controller (MVC) that takes advantage in terms of scalability, data integrity, and security. The Aftercare decision support system architecture used an object-based design approach in which is associated with uniform information extraction and the matching module. Additionally, it promises manageability and maintainability. Future researchers should conduct a similar study on the different decision tree algorithms of an aftercare decision support system.

\section{REFERENCES}

1. National Institute On Drug Abuse (2014), Treatment Approaches For Drug Addiction, Available at http://www.drugabuse.gov/ publications/ drugfacts /treatment-approachesdrugaddiction

2. Makinano, M. (2018), Rethinking PH drug policy: Points for considerations, Manila Times, Available at: https://www.pressreader.com/

3. Carcamo, D. (2015), PDEA: $92 \%$ of Metro Manila barangays drug-affected, The Philippine Star. https://www.philstar.com/nation/2015/02/19/14254 62/p dea-92-metro-manila-barangays -drugaffected

4. Raymundo, P.T. (2017) PNP resumes Tokhang to look into $1.18 \mathrm{M}$ drug surrenderees. Retrieved from

5. http://www.canadian inquirer.net/2017/03/11/pnpresumes-tokhang-to-look-into-1-18-m-drugsurrenderees/

6. Palatino, M. (2017), Duterte's 'War on illicit drugs' in the Philippines: By the numbers. The Diplomat.

7. Retrieved from http://thediplomat.com/2017/01/ dutertes -war-on-illicitdrugs-in-the-philippines-bythe-numbers/

8. Fedotov, Y. (2018) World Drug Report, United Nations Publication, Sales No. E.18.XI.9 ISBN: 978-92-1-

9. 148304-8, eISBN: 978-92-1-045058-4, Available at:

10. https://www.unodc.org/ wdr2018/prelaunch/ WDR18_Booklet_4_YOUTH.pdf

11. World Drug Report (2017), World Drug Report 2017: 29.5 million people globally suffer from drug use

12. disorders, opioids the most harmful, Available at https://www.unodc.org/unodc/en/press/releases /2017/June/world-drug-report-2017_-29-5-millionpeople-globally-suffer-from-drug-use-disorders-apioids-the-most-harmful.html 